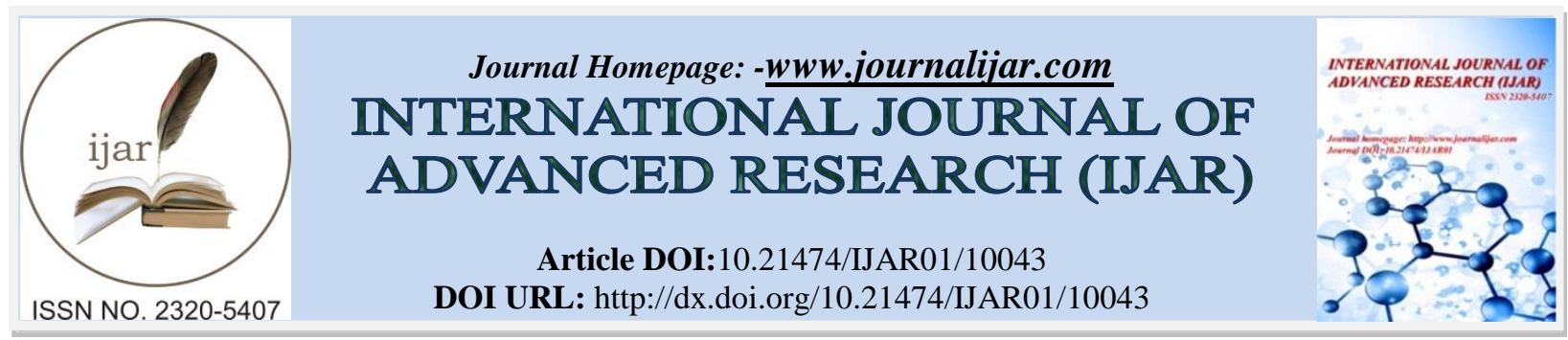

RESEARCH ARTICLE

\title{
EMERGENCE OF IMIPENEM RESISTANT ACINETOBACTER BAUMANNII ISOLATES FROM VARIOUS CLINICAL SAMPLES IN A TERTIARY CARE HOSPITAL IN KANCHIPURAM DISTRICT.
}

\section{M.Anitha ${ }^{1}$, Sumathi $\mathbf{G}^{1}$, P.A.T. Jagatheeswary ${ }^{2}$ and Nithyalakshmi Jayakumar' ${ }^{1}$}

1. Department of Microbiology, Sri Muthukumaran Medical College Hospital and Research Institute, Dr. M.G.R Medical University, Chikkarayapuram, Chennai, Tamil Nadu, India.

2. Department of Microbiology, Saveetha Institute of Medical and Technical Sciences, Saveetha University, Thandalam, Chennai, Tamil Nadu, India.

\section{Manuscript Info}

..........................

Manuscript History

Received: 10 September 2019

Final Accepted: 12 October 2019

Published: November 2019

Key words:-

Acinetobacter baumannii, antimicrobial susceptibility, clinical samples, imipenem resistance.

\begin{abstract}
Background: Acinetobacter is an important opportunistic pathogen and is a common cause of hospital acquired infections including bacteremia, pneumonia, urinary tract infection, peritonitis, etc. In the recent past carbapenems had been drugs of choice for serious infections with Acinetobacter baumannii, but imipenem resistant strains are rapidly emerging.

Objectives: The aim of the study was to determine the prevalence of imipenem resistant Acinetobacter baumannii(IRAB) isolates from various clinical samples in a tertiary care hospital.

Methods: A total of 734 samples were collected over a period of one year from November 2017 to November 2018. Bacterial isolates were identified using standard methods. Antibiotic susceptibility testing was done by Kirby Bauer disc diffusion method according to the Clinical and Laboratory Standards Institute(CLSI) guidelines.

Results: Of 734 specimens, 191 (26\%) were Acinetobacter baumannii. Among the Acinetobacter baumannii isolates 93 (49\%) were resistant to imipenem. Isolates were predominantly from males $(56 \%)$ followed by females(44\%). Maximum number of Acinetobacter baumannii were from urine samples $67(35 \%)$, followed by pus $43(23 \%)$, sputum $37(19 \%)$, miscellaneous (body fluids and others) 29(15\%) and blood 16(8\%). Imipenem Resistant Acinetobacter baumannii (IRAB)were from urine samples 31(33\%), followed by pus 22(24\%), sputum 18(19\%), miscellaneous (body fluids and others) 15(16\%) and blood $7(8 \%)$.

Conclusion: This study highlights the high prevalence of imipenem resistant Acinetobacter baumannii among clinical samples. The emergence of IRAB is a serious global threat to public health. Hence there is a need for strict infection control and monitoring of antimicrobial therapy to combat infections caused by IRAB.
\end{abstract}

Copy Right, IJAR, 2019,. All rights reserved.

Corresponding Author:-M.Anitha.

Address:-Department of Microbiology, Sri Muthukumaran Medical College Hospital and Research Institute, Dr. M.G.R Medical University, Chikkarayapuram, Chennai, Tamil Nadu, India. 


\section{Introduction:-}

Acinetobacter spp. are Gram negative, strictly aerobic, non-fastidious, non-fermenting encapsulated coccobacilli causing mostly hospital acquired infections. According to recent literature, Acinetobacter spp. are the second most common non-fermenting gram negative pathogen isolated from clinical samples after Pseudomonas aeruginosa. ${ }^{1}$

Most frequently encountered species is Acinetobacter baumannii and it is commonly associated with infections, such as bacteremia, urinary tract infection, meningitis, skin and soft tissue infections and pneumonia with high mortality rate of $30-75 \%$ in hospitalised patients. ${ }^{2}$

In the recent past carbapenems had been drugs of choice for serious infections with Acinetobacter baumannii, but carbapenem resistant strains are rapidly emerging as a potential threat. There are several factors leading to carbapenem resistance $(\mathrm{CRAB})$ in Acinetobacter baumannii, most important being the acquisition of carbapenem hydrolysing $\beta$-lactamases. Other mechanisms include the presence of mobile genetic elements, reduced expression of outer membrane proteins, altered affinity or expression of penicillin-binding proteins and multidrug efflux pumps. ${ }^{3}$

However, increased resistance to carbapenem class of antibiotics has been reported worldwide. Results from studies have reported carbapenem resistance rate of A. baumannii as 40-75 per cent throughout India. ${ }^{4}$

The main objectives of this study is to determine the prevalence of the Acinetobacter infections from various clinical samples and to investigate imipenem resistant A. baumannii strains isolated from patients in a tertiary care hospital.

\section{Methods:-}

\section{Bacterial Isolates}

This study was conducted in the Department of Microbiology, in a tertiary care hospital in Kanchipuram district, chennai. Acinetobacter baumannii isolated from various clinical samples over a period of one year (November 2017 to November 2018). Out of 734 samples, Acinetobacter baumannii was isolated from 191 clinical samples such as urine, pus, sputum, miscellaneous (body fluids and others ) and blood were collected from patients. Informed consent was obtained from the participants before starting the procedures. The samples received in the laboratory were inoculated on 5\% Sheep Blood agar and Mac Conkey agar and incubated overnight aerobically at both $37{ }^{\circ} \mathrm{C}$ and $44{ }^{\circ} \mathrm{C}$. All isolates were further processed and identified by routine microbiological and biochemical tests as per standard methodology. ${ }^{5}$

\section{Bacterial identification and purification}

In case of urine samples, the isolates were subjected to biochemical tests only if the colony count was significant $\left(>10^{5} \mathrm{CFU} / \mathrm{ml}\right)$. In order to ensure that all isolates were pure, they were cultured three times. To confirm that all these isolates belonged to Acinetobacter baumannii characteristic colonies (Non Lactose-fermenting, glistening, small mucoid colonies) were subjected to Gram stain (Gram negative coccobacilli), motility ( non-motile) and standard biochemical reactions (Catalase, Oxidase, Oxidation- fermentation test, Indole production, Methyl Red(MR), Voges-Proskauer (VP), Citrate utilization, reaction in Triple Sugar Iron medium, Mannitol Motility test, Urease activity). ${ }^{5}$

\section{Antibiotic Susceptibility of A. baumannii}

After identification by phenotypic methods, antibiotic susceptibility was performed for each isolate by the Kirby Bauer disc diffusion method on Mueller-Hinton agar using 0.5 MacFarland turbidity standard and comparing zone sizes with control strain Pseudomonas aeruginosa ATCC 27853. ${ }^{6}$ The antimicrobial agent used were carbapenem group imipenem. Carbapenem resistant was tested by using commercially available imipenem disc (10 $\mu \mathrm{g})$ by Kirby Bauer disc diffusion method. Antibiotic susceptibility results were interpreted by measuring the zone diameters produced and correlating them with the CLSI standards. ${ }^{7}$

\section{Statistical analysis:-}

The statistical analysis was performed using the SPSS version statistics 20 . The $\mathrm{Chi}^{2}\left(\mathrm{x}^{2}\right)$ test was used to compare the percentages of Acinetobacter baumannii prevalence and imipenem resistance rates from different clinical samples. The $\mathrm{p}$ values $<0.05$ were considered statistically significant. 


\section{Results:-}

During the study period, out of 734 specimens received, 191 isolates were positive for Acinetobacter baumannii (Figure 3) of which 93 isolates were imipenem resistant (Figure 4) Male patients (56\%)predominated over female patients(44\%) as depicted in the Table 1.

Table 2 \& Figure 1 shows the distribution of the isolates in various clinical samples. Maximum isolates were isolated from urine samples 67(35\%), followed by pus 43(23\%), sputum 37(19\%), miscellaneous (body fluids and others) $29(15 \%)$ and blood 16(8\%).

Among all the 191 (26\%) isolates of Acinetobacter baumannii, 93 (49\%) were resistant to imipenem by disc diffusion method. Therefore maximum imipenem resistance was observed in urine samples 31(33\%), followed by pus $22(24 \%)$ sputum 18(19\%), miscellaneous (body fluids and others) $15(16 \%)$ and blood 7(8\%) as revealed in the Table 3 \& Figure 2.

\section{Statistical analysis:-}

Data were recorded and statistical analyses were performed by SPSS version 20 software using chi-square test. Hence, $\mathrm{P}$ value $<0.05$ was considered as the statistically significance level.

Table 1:-Gender distribution of Acinetobacter baumannii from different clinical samples $(\mathbf{n}=191)$

\begin{tabular}{|l|l|l|}
\hline Gender & No of isolates & Percentage (\%) \\
\hline Male & 107 & $56 \%$ \\
\hline Female & 84 & $44 \%$ \\
\hline Total & 191 & $100 \%$ \\
\hline
\end{tabular}

Table 2:-Isolation of Acinetobacter baumannii from various samples $\mathrm{n}=191$

\begin{tabular}{|l|l|l|}
\hline Clinical samples & No of isolates & Percentage (\%) \\
\hline Urine & 67 & $35 \%$ \\
\hline Pus & 43 & $23 \%$ \\
\hline Sputum & 37 & $19 \%$ \\
\hline Miscellaneous (body fluids and others) & 29 & $15 \%$ \\
\hline Blood & 16 & $8 \%$ \\
\hline Total & $\mathbf{1 9 1}$ & $\mathbf{2 6 \%}$ \\
\hline
\end{tabular}

Table 3:- Isolation of Imipenem resistant Acinetobacter baumannii(IRAB) from various samples $\mathbf{n}=\mathbf{9 3}$

\begin{tabular}{|l|l|l|}
\hline Clinical samples & No. of isolates & Percentage (\%) \\
\hline Urine & 31 & $33 \%$ \\
\hline Pus & 22 & $24 \%$ \\
\hline Sputum & 18 & $19 \%$ \\
\hline Miscellaneous (body fluids and others) & 15 & $16 \%$ \\
\hline Blood & 7 & $8 \%$ \\
\hline Total & $\mathbf{9 3}$ & $\mathbf{4 9 \%}$ \\
\hline
\end{tabular}


Figure 1:-Distribution of A.baumannii from various clinical samples

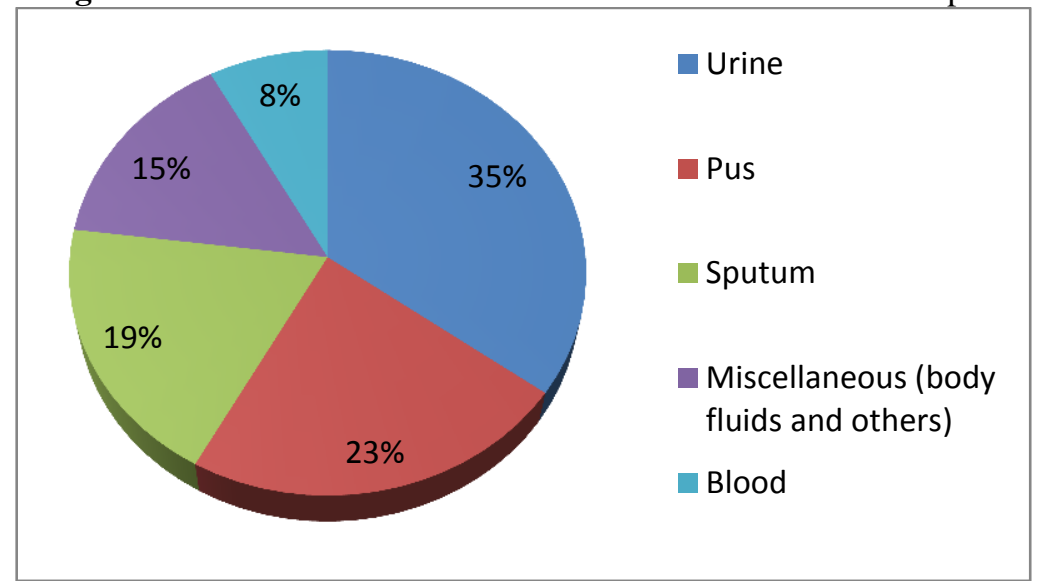

Figure 2: Distribution of IRAB from various clinical samples

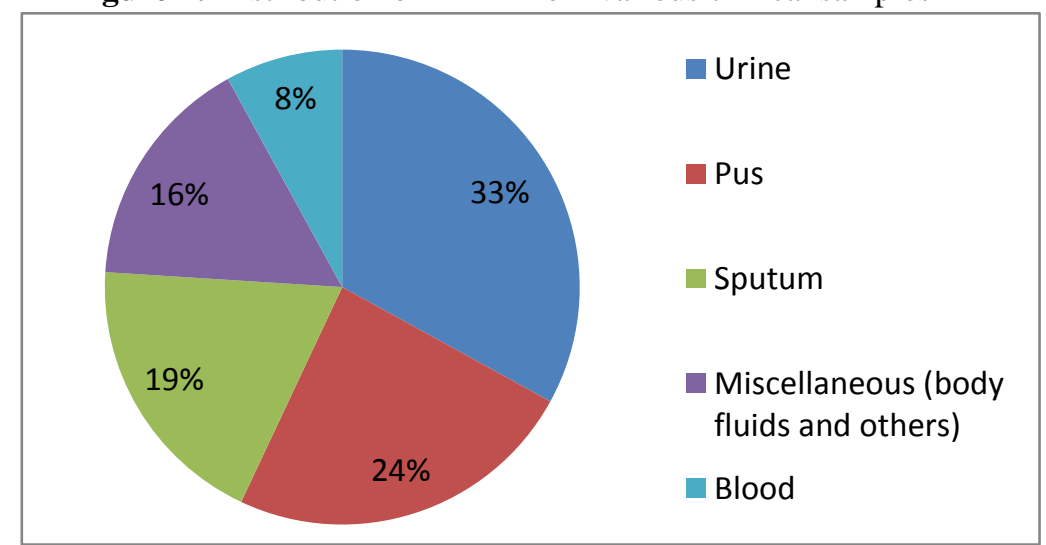

Figure 3:-Biochemical tests of A.baumannii

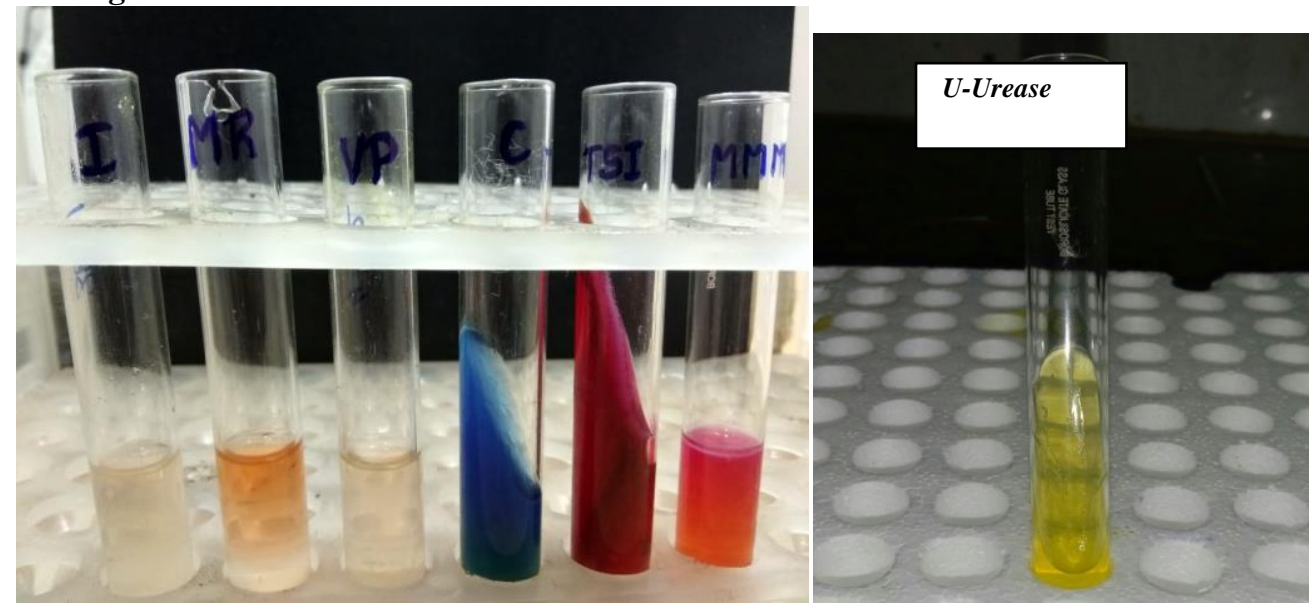

I-Indole, MR-Methyl Red, VP-Voges Proskauer, C-Citrate,

TSI-Triple Sugar Iron, MMM-Mannitol Motility Test 


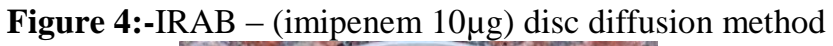

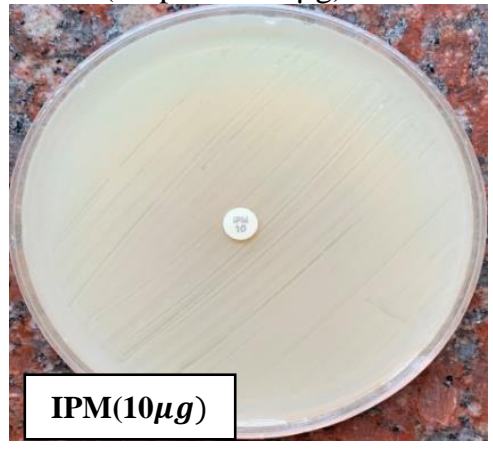

\section{Discussion:-}

In the recent years, A. baumannii infection has become a critical challenge to healthcare systems and has contributed to increased morbidity and mortality among patients in Tamil nadu. Control of A. baumannii infections is always difficult because A. baumannii is resistant to several antimicrobial agents, including imipenem, which remains as the drug of choice, even though frequency of IRAB isolates are reported to be on the rise worldwide. ${ }^{8}$

Among 191(26\%) strains, 56\% isolates were from males and 44\% from females. In correlation with this study, Nadheema et al.,(2013) ${ }^{9}$ showed A. baumannii isolates were recovered from 75 patients; $67(89 \%)$ men and $8(11 \%)$ women. In contrast to this study Anuradha et al., (2015) ${ }^{10}$ reported that Acinetobacter was isolated most commonly from females.

In the present study Acinetobacter baumannii was isolated from various clinical samples including urine, pus, sputum, miscellaneous (body fluids and others) and blood. The total percentage of distribution of Acinetobacter baumannii among the culture samples showed more distribution among urine samples (35\%) followed by pus (23\%), sputum (19\%), miscellaneous (body fluids and others) $(15 \%)$ and blood (8\%). Similar prevalence was shown in a study conducted by Lone et al (2009) ${ }^{11}$ which showed predominant distribution of Acinetobacter among urine $(39.6 \%)$ followed by pus $(29.5 \%)$.

Unlike this study, Vishnu Preyaet al. (2019) ${ }^{2}$ reported prevalence of Acinetobacter isolates from pus samples (42.5 $\%$ ) followed by urine (22.5\%), sputum (15\%), others(12.5\%), blood(5\%) and body fluids(2.5\%). Studies by Manikal et al.(2000) $)^{12}$ and Fiji E et al.,(2018) ${ }^{13}$ were in concordance with this present study showing lower isolation rates of $17 \%$ and $12.8 \%$ from blood samples.

The present study demonstrates the presence of imipenem resistant Acinetobacter baumannii (IRAB) isolates were found to be (93)49\%. This study rate is higher than those of previous studies by Anuradha et al.,(2015) ${ }^{(10)} 9.5 \%$, Gaur A et al.,(2008) ${ }^{14} 23.1 \%$ and a study by Amarjeet Kaur et al.,(2014) ${ }^{15} 40.3 \%$.

This study rate is lower than that other studies where the antibiotic resistance to imipenem was $57.4 \%$ by Kabbaj et al.,(2013), ${ }^{16} 76.19 \%$ by Jean et al.,(2016) ${ }^{17}$ and $100 \%$ resistance to imipenem by Vishnu Preya et al.,(2019) ${ }^{2}$. But these reports were not in consistent with studies by Anitha et al., (2016) ${ }^{18}$ who showed $100 \%$ sensitive to IRAB.

Current study revealed imipenem resistant A. baumannii isolated from urine, pus, sputum, miscellaneous (body fluids and others) and blood were found to be 33\%, 24\%,19\%, 16\% followed by $8 \%$. (Table 2) In a study by Nadheema et al.,(2013), ${ }^{9}$ the proportion of imipenem resistant A. baumannii (IRAB) isolates was found to be $77.27 \%$ from sputum specimens followed by $74.07 \%, 56.67 \%, 37.5 \%$ and $16.67 \%$ from wounds, blood, urine and burns specimens respectively, which is not in agreement with the present study.

\section{Conclusion:-}

Acinetobacter is a common threat in hospital acquired infections. In this study Acinetobacter baumannii were found to be $49 \%$ imipenem resistant. Emergence of carbapenem resistance is troublesome nowadays. It is a great challenge for physicians to treat Acinetobacter baumannii infections which is associated with high mortality, highlighting the need for strict infection control strategies.

To avoid antibiotic resistance, antibiotics should be used sensibly and alternative therapy should be considered for each hospital according to the resistance rates in the hospital... 


\section{Acknowledgements:-}

We acknowledge our technical staff for helping us in laboratory work and Dr.K.Siva for helping in statistics from SMMCH \&RI and special thanks to J.A.Emaya who supported to complete the project.

\section{References:-}

1. Gautam V, Singhal L, Ray P. (2011): Burkholoderia cepacia complex. Beyond Pseudomonas and Acinetobacter. Ind J Med Microbiol, 29:4-12.

2. K. Vishnu Preya and R. Nepoleon. (2019): Analysis of Carbapenem Susceptibility Pattern among Acinetobacter isolates in a Tertiary Care Hospital, india. Int.J.Curr.Microbiol. App.Sci 8(3): 1423-1429.

3. Yu YS, Yang Q, Xu XW, Kong HS, Xu GY, Zhong BY. (2004): Typing and characterization of carbapenemresistant Acinetobacter calcoaceticus-baumannii complex in a Chinese hospital. J Med Microbiol, 53: 653-6.

4. Saranya Vijayakuma, Purva Mathur, Arti Kapil et al., (2019) : Molecular characterization \& epidemiology of carbapenem-resistant Acinetobacter baumannii. Indian J Med Res, pp 240-246.

5. Tille PM. Bailey and Scott's Diagnostic Microbiology, 13th edition. (2014): St. Louis, Missouri: Elsevier, 193231.

6. Bauer AW, Kirby WM, Sherris JC, Turck M. (1966): Antibiotic susceptibility testing by a standardized single disk method. Am J Clin Pathol, 45:493-6.

7. Clinical Laboratory Standards Institutes (CLSI). (2016): Performance standards for antimicrobial susceptibility testing. 26th ed. CLSI supplement M100S. Wayne, PA: Clinical and Laboratory Standards Institute, Jan;36(1):40-256

8. Tang SS, Apisarnthanarak A, Hsu LY. (2014): Mechanisms of $\beta$-lactam antimicrobial resistance and epidemiology of major community-and healthcare-associated multidrug-resistant bacteria. . Adv Drug Deliv Rev,78:3-13.

9. Nadheema Hammood Hussein, Harith Jabbar Fahad Al-Mathkhury and Majeed Arsheed Sabbah. (2013): Imipenem-Resistant Acinetobacter baumannii isolated from patients and hospitals environment in Baghdad. Iraqi Journal of Science, Vol 54, No.4, pp:803-812.

10. Anuradha, Muthulakshmi, Somasunder.(2015): Phenotypic characterization of Acinetobacter from various clinical samples and molecular study on imipenem resistant strains in a tertiary care hospital, Enathur, Kanchipuram. European J Pharmaceutical Med Res,2(6):314.

11. Lone R, Shah A, Kadri SM, Lone S, Faisal S. (2009): Nosocomial Multi-Drug-Resistant Acinetobacter Infections - Clinical Findings. Risk Factors and Demographic Characteristics. Bangladesh J Med Microbiol, 3:34-8.

12. Manikal V M, D Landman, G Saurina, E Oydna, H Lal and J Quale. (2000): Endemic carbapenem resistant Acinetobacter species in brooklyn, New York: City wilde prevalence. Inter-institutional spread, and relation to antibiotic usage. ClinInfec Dis, 31:101-106.

13. Fiji E, B Anandharaj. (2018): Regional Prevalence and Evaluation of Carbapenem Resistant A. baumannii. International Journal of Pharmaceutical and Clinical Research, 10(9): 234-237 ISSN- 09751556.

14. Gaur A, Garg A, Prakash P, Anupurba S, Mohapatra TM. (2008): Observations on carbapenem resistance by minimum inhibitory concentration in nosocomial isolates of Acinetobacter species: an experience at a tertiary care hospital in North India. J Health Popul Nutr, 26(2): 183-8.

15. Amarjeet Kaur, Veenu Gupta, Deepinder Chhina. (2014) Prevalence of metallo- $\beta$-lactamase-producing (MBL) Acinetobacter species in a tertiary care hospital IRAN. J. Microbiol. Vol. 6, No. 1, 22-25.

16. Kabbaj H, Seffar M, Belefquih B, Akka D, Handor N, Amor M et al., (2013): Prevalence of Metallo$\beta$ Lactamases Producing Acinetobacter baumannii in a moroccan Hospital. ISRN Infectious Diseases. Article ID 154921, 3 pages www.hindawi.com/isrn/ id/2013/154921.

17. Jean Uwingabiye, Mohammed Frikh, Abdelhay Lemnouer et al.,(2016): Acinetobacter infections prevalence and frequency of the antibiotics resistance: comparative study of intensive care units versus other hospital units ThePanAfrican Medical Journal, 23:191.

18. M. Anitha, DM. Monisha, A. Mohamed Sulthan, Sathya Pandurangan. (2016): Emergence and Prevalence of Acinetobacter baumannii in Tertiary Care Hospital Settings. Sch. Acad. J. Biosci., (SAJB), 4(4A):335-341. 